\title{
Characterization and In Silico Analysis of Pregnancy-Associated Glycoprotein-1 Gene of Buffalo (Bubalus bubalis)
}

\author{
Jerome A., ${ }^{1}$ S. K. Singh, ${ }^{1}$ S. K. Agarwal, ${ }^{1}$ Mohini Saini, ${ }^{2}$ and Ashwin Raut ${ }^{3}$ \\ ${ }^{1}$ Division of Animal Reproduction, Indian Veterinary Research Institute, Izatnagar, Bareilly 243 122, India \\ ${ }^{2}$ Centre for Wildlife, Indian Veterinary Research Institute, Izatnagar, Bareilly 243 122, India \\ ${ }^{3}$ Division of Animal Biotechnology, Indian Veterinary Research Institute, Izatnagar, Bareilly 243 122, India
}

Correspondence should be addressed to Jerome A., jerome210982@gmail.com

Received 27 September 2010; Accepted 3 January 2011

Academic Editor: Meredith Yeager

Copyright ( 2011 Jerome A. et al. This is an open access article distributed under the Creative Commons Attribution License, which permits unrestricted use, distribution, and reproduction in any medium, provided the original work is properly cited.

\begin{abstract}
Pregnancy-Associated Glycoproteins (PAGs) are trophoblastic proteins belonging to the Aspartic proteinase family secreted by different placental cells of many mammalian species. They play a pivotal role in placentogenesis, foetomaternal unit remodeling, and implantation. The identification of the genes encoding those proteins will be helpful to unravel the intricate embryogenomic functions during pregnancy establishment. Considering importance of these proteins, the present study was undertaken to characterize the pregnancy associated glycoprotein-1 gene of buffalo. An 1181 base pairs buffalo PregnancyAssociated Glycoprotein PAG-1 gene was PCR amplified from the RNA obtained from the fetal cotyledons. BLAST analysis of the buffalo PAG-1 sequence retrieved a total of 20 cattle, 5 goat, and 4 sheep PAG sequences, exhibiting more than $80 \%$ similarity. Buffalo PAG-1 gene contained an uninterrupted open reading frame of 1140 base pairs encoding 380 amino acids that possess a 15 amino acid signal peptide and mature peptide of 365 amino acids. The phylogenetic study of the buffalo PAG-1 gene revealed buffalo PAG-1 is more related to cattle, goat, and sheep PAG-1 sequences. By this study characterization of buffalo PAG-1 gene and its evolutionary relationship was deduced for the first time.
\end{abstract}

\section{Introduction}

Pregnancy is established and maintained by the two-way communication between the conceptus and the mother. These intricate dialogues which are initiated after fertilization are crucial as these signals are considered potential markers for effective placental remodeling, pregnancy recognition, and successful implantation. These interactions between the conceptus and maternal system emphasize the importance of both the components in maternal recognition of pregnancy and embryonic development [1]. These important signals to the maternal system to sustain pregnancy are mediated by numerous molecules which include steroid hormones, peptide hormones, cytokines, and growth factors $[1,2]$.

Conceptus-derived substances are considered to be precise and reliable markers of pregnancy and fetal well-being. Pregnancy-associated glycoproteins are one such large family of protein molecules produced by conceptus for the recognition by the mother. Pregnancy-associated glycoproteins (PAGs) are acidic glycoprotein belonging to the Aspartic Proteinase superfamily sharing more than $50 \%$ amino acid sequence identity with Pepsin, Cathepsin D, and E $[3,4]$.

Pregnancy-associated glycoproteins (PAGs) form very large family of glycoproteins; nearly 22 different PAGs in ruminants have been identified at different stages of gestation [5]. Pregnancy-Associated Glycoprotein-1 (PAG-1) also known as Pregnancy Specific Protein B (PSPB), PSP-60, and SBU3, is secreted by the binucleate cells of the conceptus trophectoderm [6]. PAG-1 is detectable in maternal blood soon after implantation as binucleate cells migrate from the trophectoderm and fuse with uterine epithelial cells and hence it is considered as a potential signal from the conceptus [7]. The products of binucleate cells in maternal circulation have also been reported to be associated with placental mass, fetal number, twins, and neonatal birth weight in cattle $[8,9]$. 
The Pregnancy-associated glycoproteins (PAGs) are multigene family expressed in placenta of eutherian mammals and their expression varies spatially as well as temporally during gestation [10]. Multiple PAG genes have been cloned and identified in many domestic animals such as cattle, sheep [5], goat [11], pig [12], and wild ruminants' species [5]. Based on the evolutionary study and phylogenetic linkage bovine pregnancy associated glycoproteins family has been segregated as ancient (bovine PAG-2, bovine PAG-8) and modern (bovine PAG-1) [1315]. But there is no report on the characterization and phylogenetic analysis of pregnancy-associated glycoprotein1 gene of buffalo. Moreover, identification of gene encoding buffalo Pregnancy-Associated Glycoprotein-1 may provide an avenue for producing recombinant protein which will be helpful to develop diagnostics for early pregnancy diagnosis and marker for embryonic development [16]. Considering the importance of the gene in embryogenesis, the present study was designed to characterize and analyze pregnancyassociated glycoprotein-1 (PAG-1) gene phylogenetic lineage.

\section{Materials and Methods}

2.1. Sample Collection and RNA Isolation. Buffalo placentae were collected from local abattoir. The stage of pregnancy was estimated by measurement of crown-rump length. Placental cotyledons were collected from day 60 of pregnancy. Total RNA was isolated from fetal cotyledons using TRI reagent (Ambion, USA) following manufacture's instructions. The integrity of the extracted RNA was checked by agarose gel $(1 \%)$ electrophoresis and visualization of the gel under UV light after staining with ethidium bromide. The purity of the obtained RNA was checked by means of spectrophotometric readings at $\mathrm{OD}_{260} / \mathrm{OD}_{280}$.

2.2. cDNA Synthesis and Buffalo PAG-1 Gene Amplification. RNA from the fetal cotyledons was reverse-transcribed into cDNA with reverse transcriptase (Qiagen, Germany), oligo (dT) primers, and $500 \mu \mathrm{M}$ dNTPs at $37^{\circ} \mathrm{C}$ for 1 hour. On the basis of available PAG-1 sequences from cattle (GenBank Acc. No. M73962; NM 174411), goat (GenBank Acc. No. AF191326), sheep (GenBank Acc.No. M73961), pig (GenBank Acc. No. L34360), white-tailed deer (GenBank Acc. No. AY509865), and zebra (GenBank Acc.No. AF036952) buffalo gene-specific primers were designed using Primer select programme of DNA star software. The primers were forward (PAG-1/start F, 5'-GGATCCAGGAAATAAACATGAAGTG-3' and PAG1/stopR, 5' -TTACTGAAC CACTCYMAGCATTT-3' ).

PCR amplification was carried out in a total volume of $25 \mu \mathrm{L}$ of reaction mixture containing approximately $100 \mathrm{ng}$ of cDNA, 10X PCR buffer (100 Mm Tris-Hcl, pH 8.8 at $25^{\circ} \mathrm{C}, 5 \mathrm{pM}$ of forward and reverse primer of each, $2.0 \mathrm{mM}$ $\mathrm{MgCl}_{2}, 200 \mu \mathrm{M}$ dNTPs, $1.0 \mathrm{U}$ Taq DNA Polymerase. The PCR protocol involved an initial denaturation at $94^{\circ} \mathrm{C}$ for 2 minutes; 30 cycles of denaturation $\left(94^{\circ} \mathrm{C}\right.$ for 15 seconds), annealing (optimum temperature of $51.3^{\circ} \mathrm{C}$ for 15 seconds), and extension $\left(74^{\circ} \mathrm{C}\right.$ for 45 seconds); one cycle of final extension $\left(74^{\circ} \mathrm{C}\right.$ for 10 minutes). The PCR product was checked by $1 \%$ agarose gel electrophoresis.

2.3. Buffalo PAG-1 Gene Cloning. PCR amplicons were cloned in cloning vector (PTZ57R/T, InsTAclone, MBI, Fermentas) following the manufacturer's protocol. The $12 \mu \mathrm{L}$ of ligated product was directly added to $200 \mu \mathrm{L}$ competent cells, and cells were then immediately transferred on chilled ice for 5 minutes, and SOC was added. The bacterial culture was pelleted and plated on LB agarplate containing Ampicillin $(100 \mathrm{mg} / \mathrm{mL})$ added to agarplate at 1: 1000, IPTG $(200 \mathrm{mg} / \mathrm{mL})$ and X-Gal $(20 \mathrm{mg} / \mathrm{mL})$ for blue-white screening. Isolation of plasmid from was done using kit's protocol (Biochem Life sciences). Recombinant plasmids were characterized by PCR using gene-specific primers and restriction enzyme digestion.

Restriction enzyme analysis of the plasmid was carried out with Xba I (MBI, Fermentas USA) and Sma I (MBI, Fermentas USA) enzymes. Double digestion with EcoR I (MBI, Fermentas USA) and Sma I (MBI, Fermentas USA) restriction enzymes was also performed.

2.4. Sequencing Buffalo PAG-1 Gene. The plasmid containing buffalo PAG-1 cDNA was sequenced using M13 Forward and Reverse primer pair by primer walking in an automated DNA sequence (Sequence Analyzer Version 2.0, ABI Prism, Chromous Biotech, Bangalore). The sequence obtained was subjected to BLAST (http://blast.ncbi.nlm.nih.gov/Blast.cgi) to retrieve PAG-1 sequences of other species. The nucleotide and deduced amino acid sequences of buffalo PAG-1 gene were aligned and compared with other species sequences available in GenBank using Clustal option in MegAlign (Lasergene Software, DNASTAR). The buffalo PAG-1 protein structure was predicted by online SWISS MODEL software. The domain structure, glycosylation sites, and hairpin loop structure were determined by online software like PROSITE (http://www.expasy.ch/pro), SMART (http://smart.embl-heidelberg.de/). The predicted buffalo PAG-1 protein sequence was statistical analysed using SAPS software. Phylogenetic tree based on the evolutionary distances was constructed using MegAlign (Lasergene Software, DNASTAR), based on the nucleic acid and amino acid alignment. Using MEGA 4.1 software the number of synonymous substitution per synonymous site $(d S)$ and number of nonsynonymous substitution per nonsynonymous sites $(d N)$ were estimated, and neutral $(d S=d N)$, positive $(d N>d S)$, or purifying $(d N<d S)$ selections were tested with a codonbased $\mathrm{Z}$ test using Nei Gojobori method.

\section{Results and Discussion}

3.1. Buffalo PAG-1 Transcript. The concentration of RNA was checked by analyzing OD260/OD280 ratio which was found in the range of 1.8-1.9 indicated the purity of the RNA, and the yield was obtained in range of $3.5-3.8 \mu \mathrm{g} / \mathrm{mL}$. From the obtained total RNA, cDNA was synthesized and PCR amplification was carried out in 1\% agarose gel. Agarose gel electrophoresis revealed 1181 bp PCR product of buffalo 


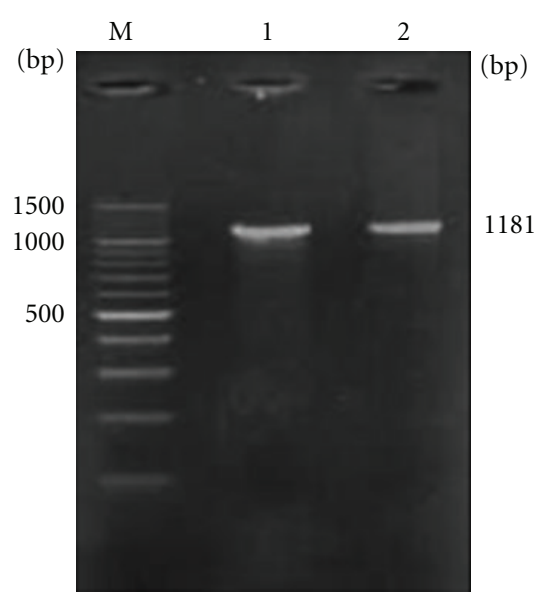

Figure 1: Agarose gel electrophoresis depicting PCR amplified Buffalo PAG-1 gene. Lanes 1 and 2: 1181 bp buffalo PAG-1 gene. Lane M: 100 bp DNA ladder as molecular size marker.

PAG-1 gene (Figure 1). Following this restriction enzyme analysis of the plasmid with Xba I and Sma I enzymes released an insert of 1181 bp. Digestion with EcoR I and Sma I released as well as digested the insert into fragments of 234 and $947 \mathrm{bp}$, respectively, confirming the presence of a conserved site for EcoR1 for every binucleate specific PAGs.

3.2. Sequence Analysis. BLAST analysis of the amplicon sequence retrieved gene sequences of various aspartic proteinase family members which showed greater than $80 \%$ similarity. Amongst these, there were 20, 5, and 4 sequences of bovine, caprine, and ovine PAG, respectively, that exhibited more than $80 \%$ similarity with buffalo PAG-1 sequence and 5 sequences of white tailed deer which showed less than 80\% homology. Accordingly, the sequence was submitted to Genbank as buffalo Pregnancy-Associated Glycoprotein1 gene under accession number EU815059. The deduced buffalo PAG-1 gene consists of an open reading frame of 1140 nucleotide corresponding to an inferred polypeptide length of 380 amino. Moreover, on translation buffalo PAG-1 gene sequence encodes a signal sequence constituting the first 15 amino acids and a mature peptide of 365 residues.

Buffalo PAG-1 gene was compared with the members of the aspartic proteinase family, Pregnancy-Associated Glycoprotein gene sequences of cattle and other species.

With the members of aspartic proteinase family buffalo PAG-1 sequence showed a similarity of 62.4, 34.6, 33.8, 32.6, 29.5 , and $28.7 \%$ with pepsinogen, cathepsin E, chymosin, pepsin, cathepsin $\mathrm{D}$, and rennin, respectively, at the amino acid level. With cattle PAG gene sequences buffalo PAG-1 sequence showed a similarity more than $80 \%$ with PAG-1, 3,16 , and less than $80 \%$ with PAG-2, 4, 5, 6, 7, 9, 14, 15, 17, $18,19,20$, and 21. Buffalo PAG-1 sequence showed highest similarity of $89.3 \%$ with cattle PAG-1 and lowest of $54.0 \%$ with cattle PAG-8.

3.3. Phylogenetic Analysis. The phylogenetic analysis of the obtained sequence of buffalo PAG-1 gene (GenBank Acc

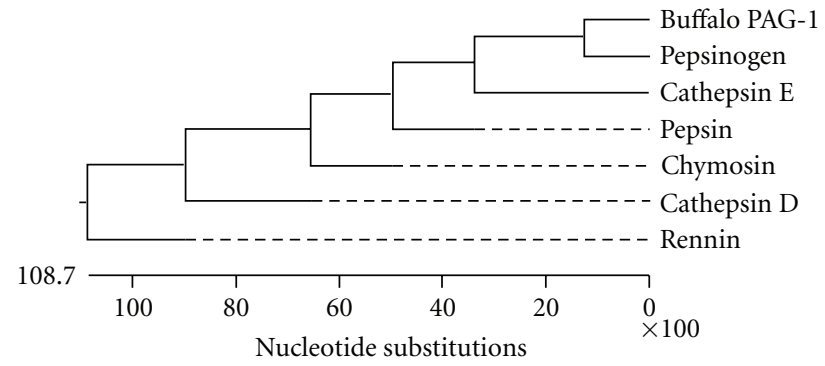

Figure 2: Phylogram depicting the evolutionary relationship of buffalo PAG-1 with members of aspartic proteinase family-based amino acid sequence.

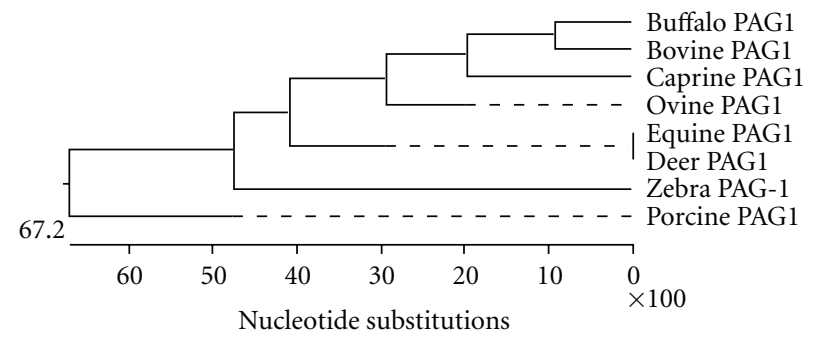

Figure 3: Phylogram depicting the evolutionary relationship of buffalo PAG-1 with other species PAG-1 sequences.

No. EU815059) with members with aspartic proteinase family members revealed that buffalo PAG-1 sequence is more related to pepsinogen and less related with rennin (Figure 2). The degree of similarity was determined comparing the obtained buffalo nucleotide and derived amino acid sequence with available PAG-1 sequences of cattle (GenBank Acc. No. M73962;.NM 17441), goat (GenBank Acc. No. AF191326), sheep (GenBank Acc. No M73961), horse (GenBank Acc. No. L38511), zebra (GenBank Acc. No AF036952), white tailed deer (GenBank Acc. No AY509865), pig (GenBank Acc. No. L34360), and cat (GenBank Acc. No. AF036953). Buffalo PAG-1 showed the percent similarity ranging from 47 to $89 \%$ and 39 to $81 \%$, at nucleotide and derived amino acids sequences levels, respectively, with other species PAG-1 sequences. Buffalo PAG-1 sequence showed highest similarity of $89.2 \%$ with cattle followed by $75.6 \%$ with goat, $74.5 \%$ sheep, $72.5 \%$ white tailed deer and $52.1 \%$ pig, $51.9 \%$ cat, $47.3 \%$ zebra, and $47.1 \%$ horse.

Phylograms constructed on the basis of nucleotide and derived amino acids sequences of buffalo PAG-1 sequence with other species showed buffalo, cattle, sheep, goat, horse, white tailed deer, zebra, feline comprise one clade, and pig comprise another. Porcine PAG-1 stands alone and represents an entirely different clade. The PAG-1 sequence of cattle and buffalo belonged to same group and showed more closeness to goat. The PAG-1 of horse, deer, and zebra seems to be an evolutionary connecting link between ruminants and other nonruminants. Horse, deer, and zebra show more evolutionary closeness to each other but far distant from ruminants (Figure 3). Analysis of phylogenetic tree constructed with buffalo PAG-1 and cattle PAG sequences 


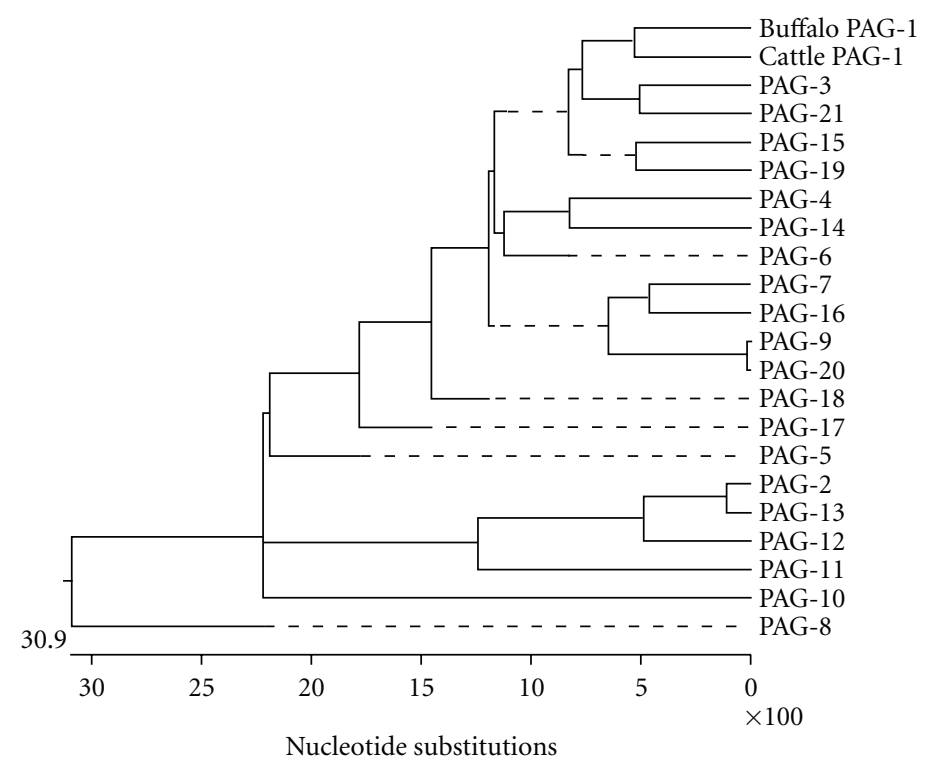

FIGURE 4: Phylogram depicting buffalo PAG-1 gene relationship with cattle PAG sequences.

revealed that buffalo PAG-1 gene is more closely related to cattle PAG-1, 3, 21, and 19 both at nucleotides and amino acids level. Cattle PAG-2 group occupies an intermediate position between the ancient aspartic proteinase family members and PAG-1 group. Buffalo PAG-1 as well as other species PAG-1 sequences comprise one clade and show more divergence from the ancient aspartic proteinase family members (Figure 4). Codon-based $\mathrm{Z}$ test using the Nei Gojobori method revealed that at 5\% level of significance, $d N$ is substantially greater than $d S$. Thus, buffalo PAG-1 might have evolved by recently by positive selection $(d N>d S)$ among these species. Buffalo and cattle PAG-1 gene showed identical lineage. Goat, sheep, horse, and deer are similar but have different lineage. Pig sequence show dissimilarities suggesting different ancestry.

3.4. Predicted Protein Analysis. The predicted buffalo PAG1 protein sequence was statistically analyzed using SAPS software revealed the total number of negatively and positively charged residues charged residues were 30 and 40, respectively. The atomic composition of the protein consists of Carbon (C) 1970; Hydrogen (H) 3011; Nitrogen (N) 521; Oxygen (O) 539; Sulfur (S) 15 with 6056 atoms. The extinction coefficient is $1.828 \mathrm{M}^{-1} \mathrm{~cm}^{-1}$, at $280 \mathrm{~nm}$ measured in water. The aliphatic index and theoretical pI is 93.32 and 9.10 , respectively, with hydropathicity index of 0.071 . The estimated half life of buffalo PAG-1 protein is 30 hours with an instability index of 32.12 predicting the protein as stable.

Sequence of deduced PAG-1 gene of buffalo revealed an insert of $1181 \mathrm{bp}$. Comparing the buffalo nucleotides and derived amino acids with available PAG-1 sequences of other species confirm buffalo PAG-1 contains an open reading frame of 1140 nucleotides which on translation corresponds to a polypeptide length of 380 amino acids similar to cattle. On translation, buffalo PAG-1 cDNA yields a polypeptide of 380 amino acids with a signal peptide encoded by the first
15 amino acids followed by a mature peptide of rest 365 residues. The signal sequence of the derived buffalo PAG1 amino acid sequence (MKWLVLLGLVAFSEC) starts and terminates with methionine and cysteine, respectively. This signal sequence is well conserved in buffalo as in bovine and other species [3] (Figure 5). The predicted buffalo PAG-1 protein structure along with its amino acid residues was also deduced (Figure 6).

The derived mature peptide sequence consists of wellconserved motif (ISF $\downarrow$ RGS) between the propeptide and the mature molecule. The arrow indicates the site of cleavage between the propeptide and the mature protein. The N-terminal ends of ruminant PAG native proteins predominantly contains the sequence of 3 amino acids, $\downarrow$ R-D/G-S which is also deduced in buffalo PAG-1-derived amino acid sequence $[17,18]$. On comparison of the amino acid sequences of buffalo PAG-1 with other species PAG sequences, it is evident that both amino terminus 91 to 98 (VVFDTGSS) and carboxyl terminus 278-284 (LVDTGTS) are conserved across the domestic species (90-100\%) suggesting importance of this region for the diagnostic function of PAG-1 and suggesting that the buffalo PAG-1 has also evolved from same family [3, 19]. Moreover, residues flanking the aspartic acid residues which are considered to be essential for catalytic activity of pepsin are well conserved in buffalo as in other domestic species [20]. Although the PAGs clearly belong to the aspartic proteinases, they are not active proteolytically owing to the key mutations close to the active site due to amino and carboxyl terminal flanking the aspartic acid residues that would likely interfere with the catalytic mechanism. On analysis it was evident that buffalo PAG-1 is not proteolytically active since it possesses key mutation of alanine substitution in place of glycine at the active site as same as boPAG-1 which will displaces a water molecule that normally resides between the two catalytic aspartic acids and is directly involved in the catalytic mechanism $[3,20]$. 


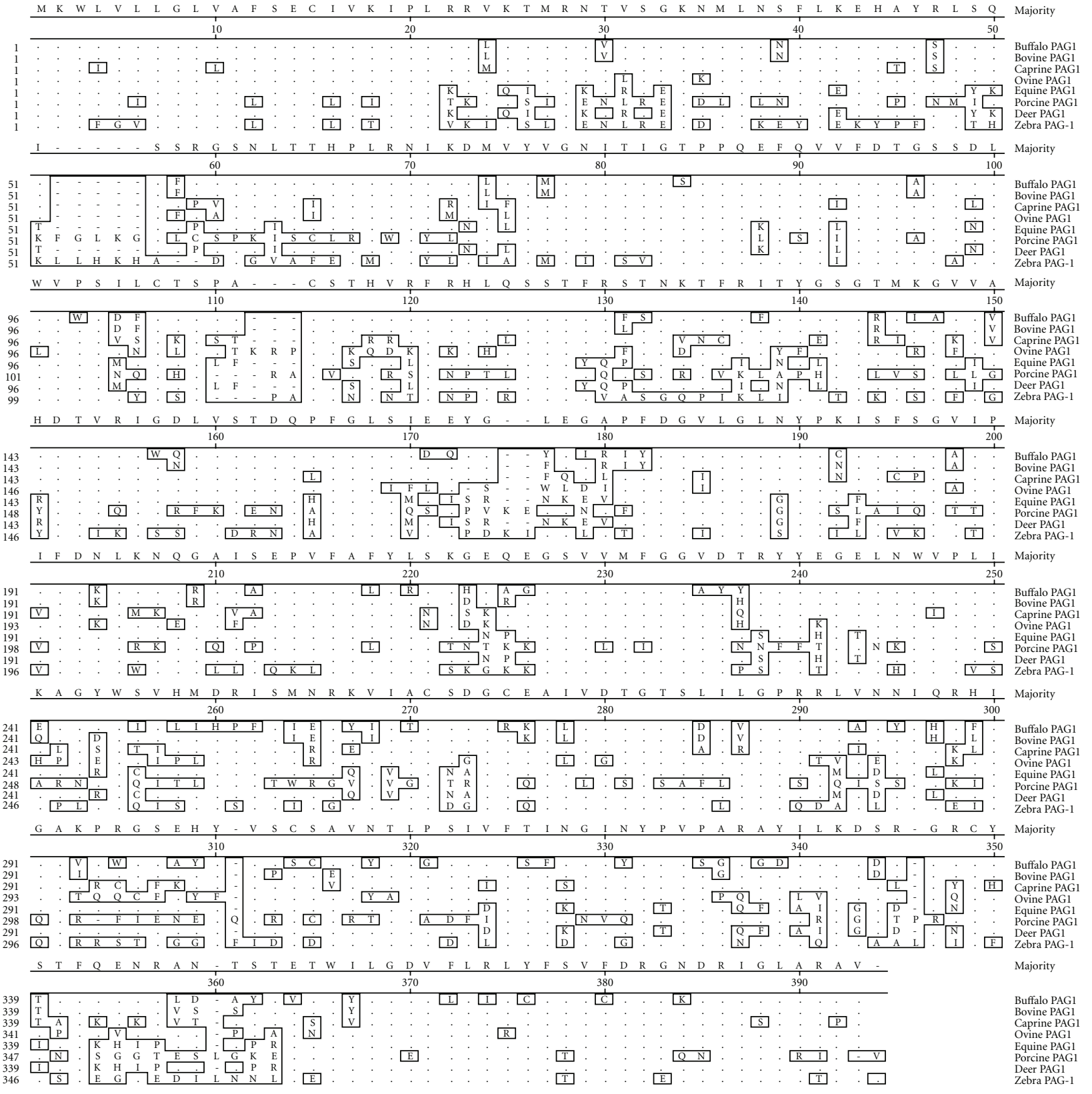

FIGURE 5: MegAlign report of buffalo PAG-1 amino acid sequence (380 amino acids) with PAG-1 amino acid sequences of other species.

Although PAG-1 group molecules are not proteolytically active, they have retained the characteristic bilobed structure of aspartic proteinase [20]. The catalytic mechanism, which leads to peptide bond cleavage in the middle of the bound peptide, is initiated through nucleophilic attack by a hydroxyl ion supplied by a water molecule strategically positioned between the two aspartic acids [19]. Comparison of buffalo PAG sequence and other species sequence reveals segments of primary structure that are hypervariable and others that are relatively constant. The region between residues 70 and 100 (13-42 pepsin numbering) showed very few substitutions depicting the presence of other conserved sequence [4]. From the alignment study it was evident that buffalo PAG-1 which showed more similarity to cattle PAG-1 will also possess a three-dimensional fold and may have a general affinity for binding peptides with basic residues [20].

The deduced buffalo PAG-1 amino acid sequence contains microsequences YS (position 46,47), LSQISF (position 48-58), RGSNLTTH (position 59-66), PLRN (position 6770), and IKDLVYMGNITIGTP (position 71-81) which are found to be conserved across ruminant PAG proteins. In general, the conserved regions are ones that are internal and structurally important for retaining the overall threedimensional fold of the molecule. By contrast, the hypervariable regions are exposed and generally correspond with surface loops. Though the physiological significance of these 


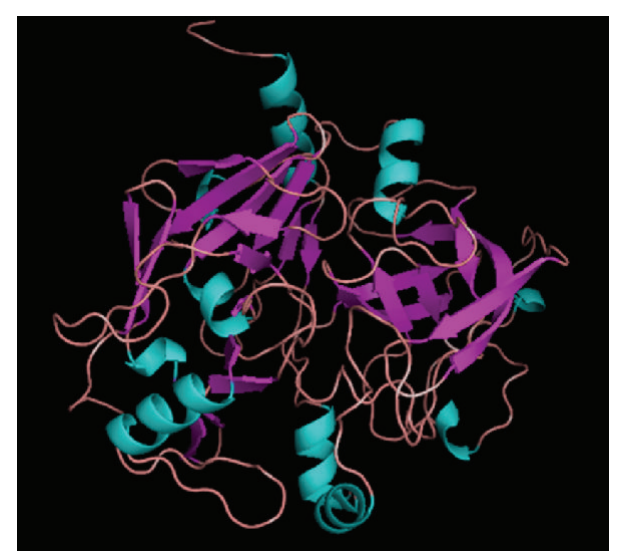

Figure 6: Predicted 3D structure of buffalo PAG-1 protein and its residues.

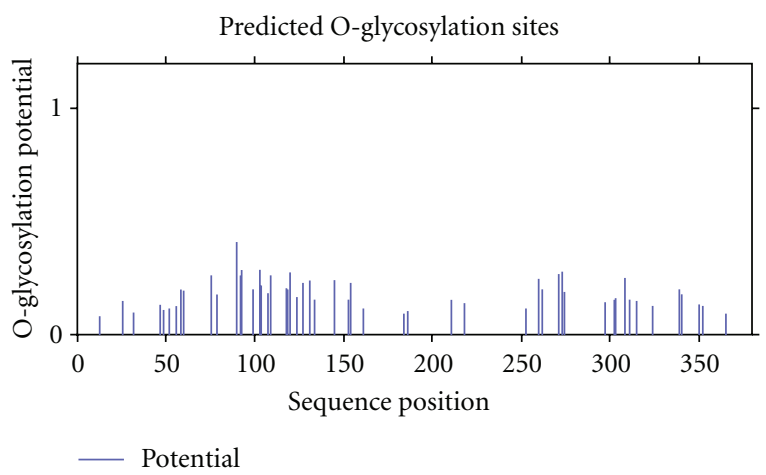

Figure 7: Predicted potential O-glycosylation sites of buffalo PAG-1 protein sequence.

changes is presently unknown, these hypervariable regions represent surface domains where amino acid substitutions could occur with little threat to the structural integrity of the molecules $[4,19]$. The buffalo mature peptide sequence also contains variable regions as in other species having profound nonsynonymous substitutions. There are mainly four variable regions at positions 101-117, 162-173, 241248 , and 331-350. Since these proteins belong to glycoprotein family, they possess distinct sites for glycosylation. The potential sites for glycosylation in deduced buffalo PAG-1 protein sequence are at positions 78-81, 127-129, and 250$300[4,20,21]$ (Figure 7).

Phylogenetic Analysis of the buffalo PAG-1 sequences with other domestic species revealed buffalo PAG-1 is more related with bovine, caprine, and ovine species and less similar to equine, porcine, deer, and zebra due to various nonsynonymous substitutions in the entire sequence in the latter species. Porcine PAG-1 forms an entire different clade as it consists of variant amino acids residues when compared with ruminant species. Phylogram constructed between buffalo PAG-1 and different bovine PAGs sequences revealed buffalo PAG-1 was more related with bovine PAG-1 thereby belonging to the recently duplicated PAG gene group (PAG-1 group) and differing significantly from the ancient PAG group consisting of PAG-2 and 8 [13-15].

\section{Conclusion}

Buffalo Pregnancy-Associated Glycoprotein-1 cDNA encoding buffalo PAG-1 protein of 380 amino acids was characterized and found to be recently duplicated group members of the aspartic proteinase family being proteolytically inactive due to key mutations close to the active site.

\section{Acknowledgments}

The authors are grateful to the Director of the Indian Veterinary Research Institute, and Head of the Animal Reproduction Division for providing the necessary facilities for conducting the experiment. The financial support by ICAR, New Delhi, India under the National Fund for Basic and Strategic Research in Agricultural Sciences (NFBSRA) project is also acknowledged.

\section{References}

[1] R. M. Roberts, Y. Chen, T. Ezashi, and A. M. Walker, "Interferons and the maternal-conceptus dialog in mammals," Seminars in Cell and Developmental Biology, vol. 19, no. 2, pp. 170-177, 2008.

[2] L. A. Salamonsen, "Role of proteases in implantation," Reviews of Reproduction, vol. 4, no. 1, pp. 11-22, 1999.

[3] N. M. Sousa, A. Ayad, J. F. Beckers, and Z. Gajewski, "Pregnancy-associated glycoproteins (PAG) as pregnancy markers in the ruminants," Journal of Physiology and Pharmacology, vol. 57, supplement 8, pp. 153-171, 2006.

[4] S. Xie, B. G. Low, R. J. Nagel et al., "Identification of the major pregnancy-specific antigens of cattle and sheep as inactive members of the aspartic proteinase family," Proceedings of the National Academy of Sciences of the United States of America, vol. 88, no. 22, pp. 10247-10251, 1991.

[5] S. Xie, B. G. Low, R. J. Nagel, J. F. Beckers, and R. M. Roberts, "A novel glycoprotein of the aspartic proteinase gene family expressed in bovine placental trophectoderm," Biology of Reproduction, vol. 51, no. 6, pp. 1145-1153, 1994.

[6] S. Xie, J. Green, B. Bao et al., "Multiple pregnancy-associated glycoproteins are secreted by Day 100 ovine placental tissue," Biology of Reproduction, vol. 57, no. 6, pp. 1384-1393, 1997.

[7] J. M. Garbayo, B. Serrano, and F. Lopez-Gatius, "Identification of novel pregnancy-associated glycoproteins (PAG) expressed by the peri-implantation conceptus of domestic ruminants," Animal Reproduction Science, vol. 103, no. 1-2, pp. 120-134, 2008.

[8] J. E. Butler, W. C. Hamilton, R. G. Sasser, C. A. Ruder, G. M. Hass, and R. J. Wiliams, "Detection and partial characterization of two bovine pregnancy-specific proteins," Biology of Reproduction, vol. 26, no. 5, pp. 925-933, 1982.

[9] A. P. Zoli, J. F. Beckers, P. Wouters-Ballman, J. Closset, P. Falmagne, and F. Ectors, "Purification and characterization of a bovine pregnancy-associated glycoprotein," Biology of Reproduction, vol. 45, no. 1, pp. 1-10, 1991.

[10] K. Klisch, N. M. De Sousa, J. F. Beckers, R. Leiser, and A. Pich, "Pregnancy associated glycoprotein-1, -6, -7, and -17 are major products of bovine binucleate trophoblast giant cells at midpregnancy," Molecular Reproduction and Development, vol. 71, no. 4, pp. 453-460, 2005. 
[11] R. G. Sasser, C. A. Ruder, K. A. Ivani, J. E. Butler, and W. C. Hamilton, "Detection of pregnancy by radioimmunoassay of a novel pregnancy-specific protein in serum of cows and a profile of serum concentrations during gestation," Biology of Reproduction, vol. 35, no. 4, pp. 936-942, 1986.

[12] O. V. Patel, I. Domeki, N. Sasaki et al., "Effect of fetal mass, number and stage of gestation on pregnancy-specific protein B concentrations in the bovine," Theriogenology, vol. 44, no. 6, pp. 827-833, 1995.

[13] M. I. Vasques, A. E. M. Horta, C. C. Marques, R. G. Sasser, and P. Humblot, "Levels of bPSPB throughout single and twin pregnancies after AI or transfer of IVM/IVF cattle embryos," Animal Reproduction Science, vol. 38, no. 4, pp. 279-289, 1995.

[14] J. M. Garbayo, J. A. Green, M. Manikkam et al., "Caprine pregnancy-associated glycoproteins (PAG): their cloning, expression, and evolutionary relationship to other PAG," Molecular Reproduction and Development, vol. 57, no. 4, pp. 311-322, 2000.

[15] B. Szafranska, S. Xie, J. Green, and R. M. Roberts, "Porcine pregnancy-associated glycoproteins: new members of the aspartic proteinase gene family expressed in trophectoderm," Biology of Reproduction, vol. 53, no. 1, pp. 21-28, 1995.

[16] S. M. Rawn and J. C. Cross, "The evolution, regulation, and function of placenta-specific genes," Annual Review of Cell and Developmental Biology, vol. 24, pp. 159-181, 2008.

[17] B. P. V. L. Telugu, A. M. Walker, and J. A. Green, "Characterization of the bovine pregnancy-associated glycoprotein gene family-analysis of gene sequences, regulatory regions within the promoter and expression of selected genes," BMC Genomics, vol. 10, article 185, 2009.

[18] O. V. Patel, T. Takahashi, K. Imai, and K. Hashizume, "Generation and purification of recombinant bovine pregnancy associated glycoprotein," Veterinary Journal, vol. 168, no. 3, pp. 328-335, 2004.

[19] A. L. Hughes, J. A. Green, J. M. Garbayo, and R. M. Roberts, "Adaptive diversification within a large family of recently duplicated, placentally expressed genes," Proceedings of the National Academy of Sciences of the United States of America, vol. 97, no. 7, pp. 3319-3323, 2000.

[20] J. A. Green, S. Xie, and R. Michael Roberts, "Pepsin-related molecules secreted by trophoblast," Reviews of Reproduction, vol. 3, no. 1, pp. 62-69, 1998.

[21] K. Guruprasad, T. L. Blundell, S. Xie et al., "Comparative modelling and analysis of amino acid substitutions suggests that the family of pregnancy-associated glycoproteins includes both active and inactive aspartic proteinases," Protein Engineering, vol. 9, no. 10, pp. 849-856, 1996. 

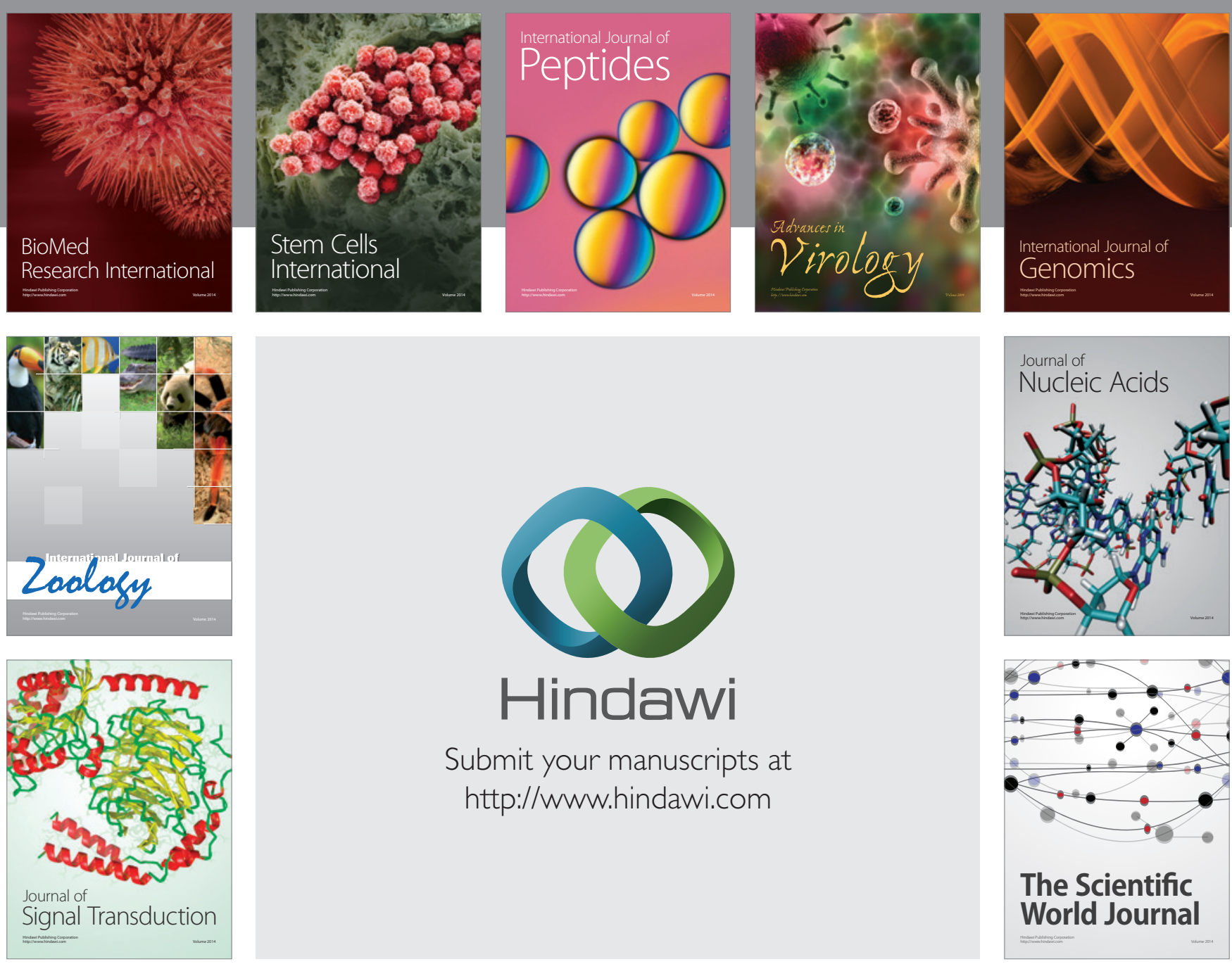

Submit your manuscripts at

http://www.hindawi.com
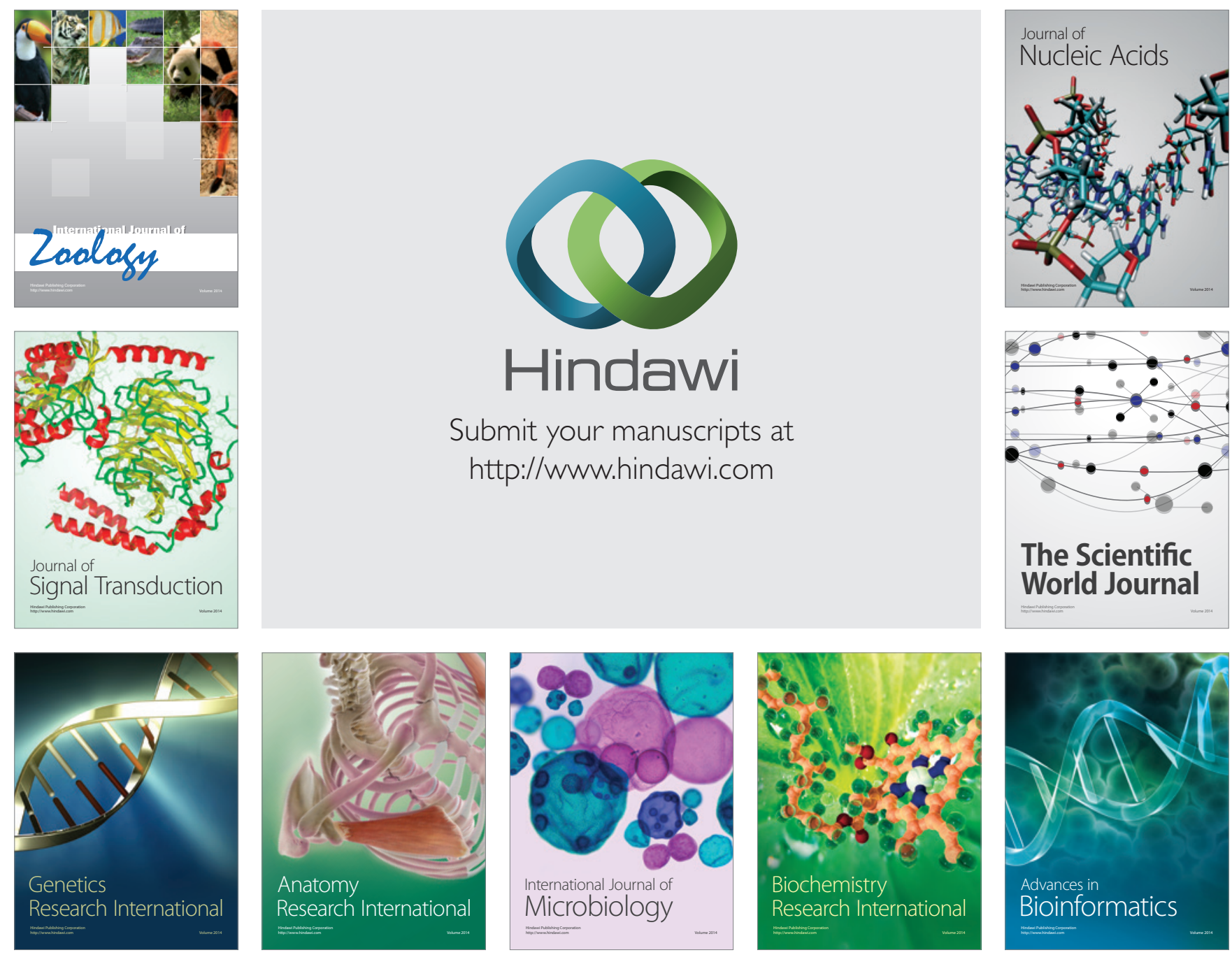

The Scientific World Journal
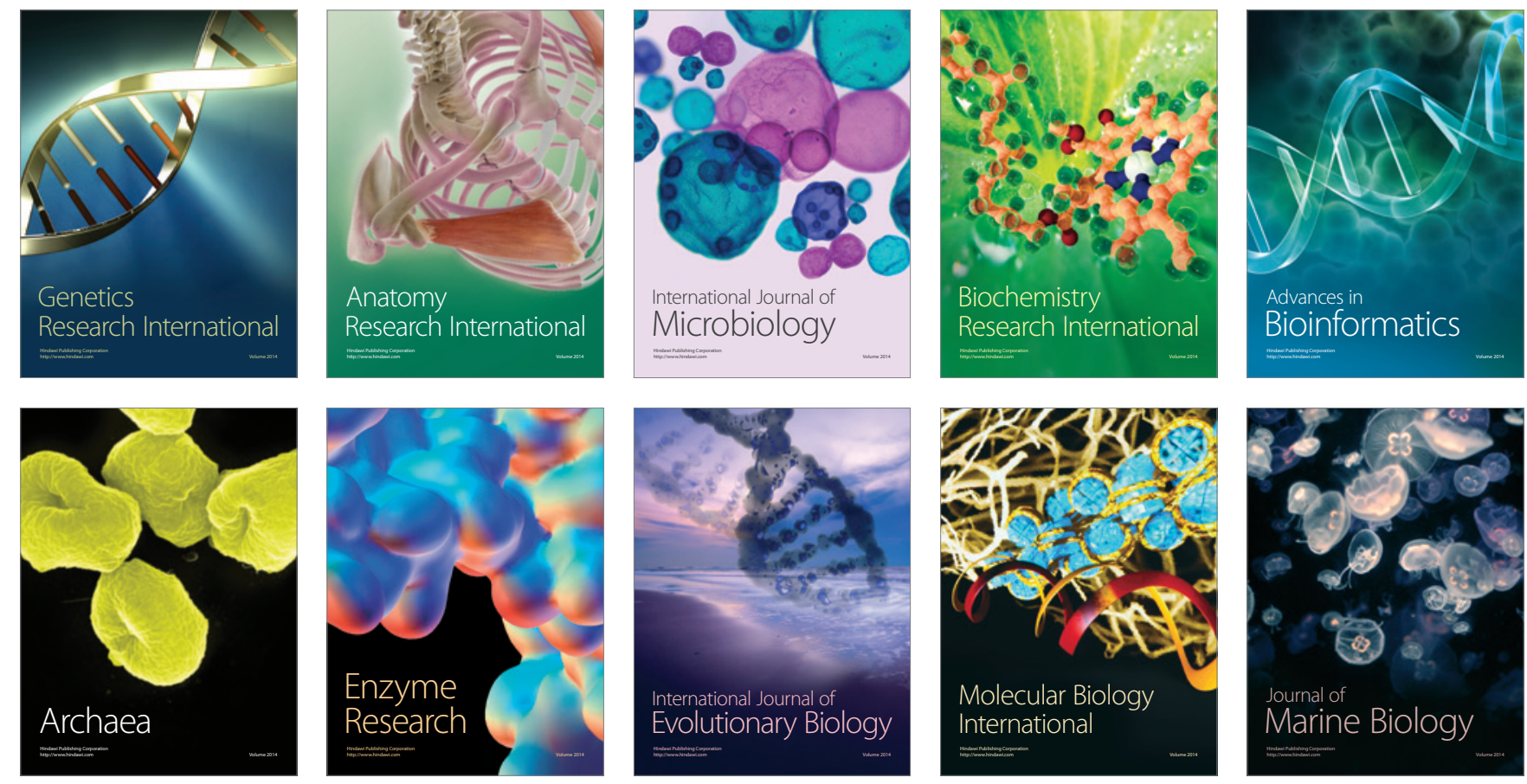